\title{
ON THE DISTRIBUTION OF THE ZEROS OF THE RIEMANN ZETA FUNCTION IN SHORT INTERVALS
}

\author{
BY AKIO FUJII ${ }^{1}$
}

\author{
Communicated by Paul Bateman, June 18, 1974
}

Here we are concerned with the distribution of the zeros of the Riemann zeta function $\zeta(s)$ in short intervals in the vertical direction. We establish a mean value theorem for the number of the zeros in short intervals and derive several consequences from it, in particular, about the difference between the ordinates of the zeros and properties of uniform distribution of the zeros. Details will appear later.

We start from the Riemann-von Mangoldt formula for the number $N(t)$ of the zeros of $\zeta(s)$ in $0<\operatorname{Im} s \leqslant t, 0<\operatorname{Re} s<1$, where the possible zeros on $\operatorname{Im} s=t$ are counted with weight one half:

$$
N(t)=L(t)+S(t) \text { for } t>t_{0},
$$

where

$$
L(t)=\frac{t}{2 \pi} \log t-\frac{1+\log 2 \pi}{2 \pi} t+\frac{7}{8}+O\left(\frac{1}{t}\right) .
$$

Here

$$
S(t)=(1 / \pi) \arg \zeta\left(\frac{1}{2}+i t\right),
$$

where $\arg \zeta(1 / 2+i t)$ is defined by continuous variation on the half line $\sigma+$ it, $\sigma \geqslant 1 / 2$ starting with the value zero if $t$ is not the ordinate of a zero of $\zeta(s)$. If the path crosses a zero, we put

$$
\arg \zeta(1 / 2+i t)=1 / 2\{\arg \zeta(1 / 2+i(t+0))+\arg \zeta(1 / 2+i(t-0))\}
$$

(cf. $[5,9.2]$.$) . Then the number$

$$
\Delta_{h} N(t) \equiv N(t+h)-N(t)=L(t+h)-L(t)+S(t+h)-S(t)
$$

of the zeros of $\zeta(s)$ in a short interval $t<\operatorname{Im} s \leqslant t+h$ essentially depends

AMS (MOS) subject classifications (1970). Primary 10H05, $10 \mathrm{H} 10$.

Key words and phrases. Riemann zeta function, distribution of zeros.

1Supported in part by National Science Foundation grant GP 36418X1.

Copyright $\odot$ 1975, American Mathematical Society 
on $S(t+h)-S(t)$. We can show, by modifying A. Selberg's approach (cf. [3]):

LEMma. For $T>1$ and $h$ in $0<h \leqslant T-T^{1 / 16}$, we have

$$
\begin{aligned}
\int_{0}^{T}(S(t+h)-S(t))^{l} d t= & \frac{2 k !}{(2 \pi)^{2 k} k !} T(2 \log (3+h \log T))^{k} \\
& +O\left((A k)^{4 k} T \cdot(\log (3+h \log T))^{k-1 / 2}\right) \text { for } l=2 k \\
= & O\left((A k)^{3 k} T(\log (3+h \log T))^{k-1}\right) \text { for } l=2 k-1 .
\end{aligned}
$$

Hence we get a mean value theorem for $N(t+h)-N(t)$ also.

An immediate consequence of this is a result about the normal density of the zeros in short intervals.

THEOREM 1. If $h \log T$ tends to $\infty$ as $T$ tends to $\infty$, then

$$
\frac{(h / 2 \pi) \log T}{\Phi(T)}<N(t+h)-N(t)<\Phi(T) \frac{h}{2 \pi} \log T
$$

for any positive increasing function $\Phi(T)$ which tends to $\infty$ as $T$ tends to $\infty$ and for almost all (in the sense of the density) $t$ in $0<t \leqslant T$.

For shorter intervals we can show

THEOREM 2.

$$
N\left(t+\frac{2 \pi C}{\log T}\right)-N(t)>C+C_{1}(\log C)^{1 / 2}(\log \log C)^{1 / 2+\epsilon}
$$

for a positive proportion of $t$ in $(0, T)$, and

$$
N\left(t+\frac{2 \pi C}{\log T}\right)-N(t)<C-C_{1}(\log C)^{1 / 2}(\log \log C)^{1 / 2+\epsilon}
$$

for a positive proportion of $t$ in $(0, T)$, where $\epsilon>0, C>C_{0}, C_{0}$ and $C_{1}$ are suitable positive absolute constants.

If we denote the ordinate of the $n$th zero of $\zeta(s)$ by $\gamma_{n}$, where $0 \leqslant$ $\gamma_{n} \leqslant \gamma_{n+1}$, the following is a consequence of Theorem 2 .

COROllaRY. For each $r=1,2,3, \cdots$ a positive proportion of $\gamma_{n}$ 's satisfy

$$
\frac{\gamma_{n+r}-\gamma_{n}}{r} \frac{\log \gamma_{n}}{2 \pi}<1-A
$$

and also a positive proportion of $\boldsymbol{\gamma}_{n}$ 's satisfy 


$$
\frac{\gamma_{n+r}-\gamma_{n}}{r} \frac{\log \gamma_{n}}{2 \pi}>1+A,
$$

where $A$ is a positive absolute constant less than 1 which may depend on $r$. In fact we can take $A=\exp \left(-C_{2} r^{2}\right)$ with a positive absolute constant $C_{2}$.

Also from our Lemma we see

THEOREM 3.

$$
\sum_{0<\gamma_{n}<T ; \gamma_{n+1}-\gamma_{n}>h}\left(\gamma_{n+1}-\gamma_{n}\right) \leqslant A \frac{T \log (3+h \log T)}{(h \log T)^{2}}
$$

uniformly for a positive $h$, and

$$
\sum_{0<\gamma_{n}<T}\left(\gamma_{n+1}-\gamma_{n}\right)^{2} \leqslant A \frac{T}{\log T}
$$

where $A$ 's are positive absolute constants.

About the property of uniform distribution of the $\gamma_{n}$ 's we can show

THEOREM 4. If $h \log T$ tends to $\infty$ as $T$ tends to $\infty$, then

$$
\sum_{m=0}^{[T / h]}\left(\Delta_{\alpha h}-\alpha \Delta_{h}\right) N(m h)=o(N(T))
$$

for each $\alpha$ in $0<\alpha \leqslant 1$.

In particular, if $h=1$, this implies $\left\{\gamma_{n}: n=1,2, \cdots\right\}$ is uniformly distributed mod 1.

From a mean value theorem of $\Delta_{h} N(t)$, we can get some information about the multiplicities of zeros. Namely, we can show

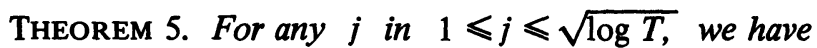

$$
\sum_{j=1}^{\infty} j^{2 k} N_{j}(T) \leqslant C N(T) \text { for } T>T_{0},
$$

where $N_{j}(T)$ is the number (counted without multiplicity) of zeros of $\zeta(s)$ in $0<\operatorname{Im} s \leqslant T, 0<\operatorname{Re} s<1$ with the multiplicity exactly $j$.

From this we see

COROllary.

$$
\varlimsup_{T \rightarrow \infty} \frac{1}{N(T)} \sum_{j=K}^{\infty} j N_{j}(T) \leqslant e^{-A \sqrt{K}}
$$

for $K>K_{0}, A$ and $K_{0}$ are suitable positive absolute constants. 
Finally we may remark here that the results in this paper have been extended to Dirichlet $L$-functions and also to Dirichlet series attached to cusp forms under certain hypotheses. $q$-analogues of this paper have been also obtained. These will also appear later.

ACKNOWLedgement. The author would like to express his gratitude to Professor P. X. Gallagher. Thanks are also due to Professor H. L. Montgomery and Professor A. Selberg.

\section{REFERENCES}

1. P. D. T. A. Elliot, The Riemann zeta function and coin-tossing, J. Reine Angew. Math. 154 (1972), 100-109. MR 47 \#1761.

2. H. L. Montgomery, The pair correlation of zeros of the zeta function, Proc. Sympos. Pure Math., vol. 24, Amer. Math. Soc., Providence, R. I., 1973, pp. 181-193.

3. A. Selberg, Contributions to the theory of the Riemann zeta-function, Arch. Math. Naturvid. 48 (1946), 89-155. MR 8, 567.

4. - The zeta-function and the Riemann hypothesis, C. R. Dixième Congrès Math. Scandinaves, 1946, Jul. Gjellerups Forlag, Copenhagen, 1947, pp. 187-200.

MR 8, 446.

5. E. C. Titchmarsh, The theory of the Riemann zeta function, Clarendon Press, Oxford, 1951. MR 13, 741.

DEPARTMENT OF MATHEMATICS, RIKKYO UNIVERSITY, TOKYO, JAPAN 